\title{
Delay Estimation Based on Compressed Sensing Subspace Pursuit Algorithm
}

\author{
Xue-dong LENG ${ }^{1,{ }^{*}}$, Zi-lun $\mathrm{ZHAO}^{2}$ and Bin $\mathrm{BA}^{1}$ \\ ${ }^{1}$ No.62, Kexue Road, Gaoxin District, Zhengzhou City, Henan Province, China \\ ${ }^{2}$ No.5, Zhongguancun South Street, Haidian District, Beijing City, China
}

Keywords: Sparse reconstruction, Delay estimation, Measurement matrix, Subspace pursuit.

\begin{abstract}
In this paper, a novel method is constructed to estimate the time delay. The purpose of this article is to deal with the lack of measurement data in small sample (single snapshot) and low signal to noise ratio environment during wireless location. First, the sparse representation model of received signals is established. And then the measurement matrix is proofed to achieve the restricted isometry property. The idea of subspace pursuit is to find the subspace which consist of the received signal. Therefore, the delay estimation can be achieved using the corresponding relation between the time delay and the measurement matrix. Finally, simulations show that the subspace pursuit algorithm is suitable for small sample environment. The method can achieve a higher precision than greedy algorithms such as orthogonal matching pursuit and Regularized orthogonal matching pursuit algorithm. Furthermore, the subspace pursuit algorithm has a better performance in anti-multi channels.
\end{abstract}

\section{Introduction}

The delay estimation is widely applied to radar, sonar, wireless location [1] and other fields, which is the research emphasis on this field in complex environment. In order to solve the insufficiency of the current algorithm in the conditions of small sample and the low signal-to-noise ratio, many new methods are introduced into the delay estimation problem. The classification method of rooting multiple signal is applied to the two-dimension parameter estimation to solve the parameter directly so that the arrival time of the pulse wireless system is achieved with combined estimation for the arrival direction, which the performance is still qualified in the condition of low signal-to-noise ratio, but the method require smooth frequency domain, so the estimation accuracy is reduced much in the condition of single-snapshot [2]. The Markov chain Monte Carlo method is applied to the passive radar location, which the mean square error still approaches the CRB in the conditions of single-snapshot and low signal-to-noise ratio, but this method is easily into stable condition in process of Markov chain sampling [3]. At the same time, that the compressed-sensing sparse-reconstruction method was proposed in recent years is widely applied to the signal processing field [4]. After processing of the time domain interference elimination and related issues, the DOA super resolution estimation is obtained by sparse-reconstruction method in the condition of single-snapshot [5]. The optimal sparse model of equal sine space based on source signal is proposed, in which the signal reconstruction and DOA estimation are achieved [6]. The measurement matrix obtained from the decomposition of Gauss random matrix's orthogonal triangle and the reception matrix of low-dimensional data obtained from the singular value decomposition are reconstructed by the formed algorithm based on compression of sensing beam, which 
the high resolution DOA estimation is achieved [7]. Single channel and multi-channel delay estimations in the condition of knowing sending signal are achieved by using orthogonal matching pursuit (OMP) algorithm, and then the sparse reconstruction method is introduced into the delay estimation, but the method is provided with a certain probability to select suboptimum atom that will cause incorrect estimation [8]. In [9], the regularized orthogonal matching pursuit (ROMP) algorithm is proposed to improve the style of choosing atoms. The compressed sensing subspace pursuit (CS-SP) algorithm can decrease the disadvantage in choosing atoms by updating the subspace that the signal is located in [10].

In the condition of knowing sending signal, one receiving signal model is constructed here based on the sparse representation in the article, and then the steering vector matrix is constructed to the measurement matrix according to the model, finally the CS-SP algorithm is used to reconstruct the gain coefficient vector. The delay unbiased estimation is obtained by one to one correspondence between the gain coefficient vector and the delay. Compare the three algorithms of the CS-SP algorithm, OMP algorithm and ROMP algorithm by the simulation experiment, which the result shows the superiority of the CS-SP algorithm applied in this article.

\section{Mathematical Model and Estimation Principle}

\section{Mathematical Model}

If the signal sent by radiating source is $s(t)$, considering of the multi-channel propagation effect of the wireless channel, the multi-channel is $L_{p}$, and the received signal is:

$$
y(t)=\sum_{i=0}^{L_{p}-1} a_{i} s\left(t-\tau_{i}\right)+n(t)
$$

In the formula, $a_{i}$ is the gain coefficient of various channels, and $n(t)$ is the additive Gauss white noise. The discrete Fourier transform is conducted for the received signal, which the frequency domain can be expressed as:

$$
Y(k)=\sum_{i=0}^{L_{p}-1} a_{i} S(k) e^{-j 2 \pi\left(f_{c}+k \Delta f\right) \tau_{i}}+N(k)
$$

In the formula, $k=0,1, \cdots K-1$ is the carrier frequency; $\Delta f$ is the frequency sampling interval; $N(k)$ means that the equalizing value is 0 ; the additive Gauss white noise with mean square deviation of $\sigma^{2}$ is recorded as $N(k) \sim \mathrm{N}\left(0, \sigma^{2}\right)$. That convert the (2) formula to vectorial form is:

$\boldsymbol{Y}=\boldsymbol{S V a}+\boldsymbol{N}$

In the formula,

$$
\begin{aligned}
& \boldsymbol{Y}=[y(0), y(1), \cdots, y(K-1)]^{\mathrm{T}} \\
& \boldsymbol{S}=\operatorname{diag}[s(0), s(1), \cdots, s(K-1)]
\end{aligned}
$$




$$
\begin{aligned}
& \boldsymbol{V}=\left[\boldsymbol{v}\left(\tau_{0}\right), \boldsymbol{v}\left(\tau_{1}\right), \cdots, \boldsymbol{v}\left(\tau_{L_{p}-1}\right)\right] \\
& \boldsymbol{v}\left(\tau_{i}\right)=\left[1, e^{-j 2 \pi \Delta \tau_{i}}, \cdots, e^{-j 2 \pi(K-1) \Delta f \tau_{i}}\right]^{\mathrm{T}} \\
& \boldsymbol{a}=\left[a_{0} e^{-j 2 \pi f_{c} \tau_{0}}, a_{1} e^{-j 2 \pi f_{c} \tau_{1}}, \cdots, a_{L_{p}-1} e^{-j 2 \pi f_{c} \tau_{L_{p}-1}}\right]^{\mathrm{T}} \\
& \boldsymbol{N}=[n(0), n(1), \cdots, n(K-1)]^{\mathrm{T}}
\end{aligned}
$$

The formula (4) is discrete Fourier transform of $y(t)$; (5) is the vector of radiating source signal; (6) and (7) are steering vectors; The formula (8) is the gain coefficient vector; The formula (9) is the additive Gauss white noise vector.

\section{Estimation Principle}

Conduct the delay estimation by using the method of sparse reconstruction, which the sparse representation of the signal is the premise. The rational construction of the measurement matrix and the design of the signal reconstruction algorithm are the core of correct estimation. Fig. 1 is the flow graph of delay estimation based on sparse reconstruction.

In the model of wireless location, the signal can be represented as the sparse form by selecting suitable sparse representation matrix. Measure the wireless multi-channel based on the time domain, the propagation delay is various with various channels and the delay representation in the time domain is sparse, so that it can be directly constructed to the form of sparse signal.

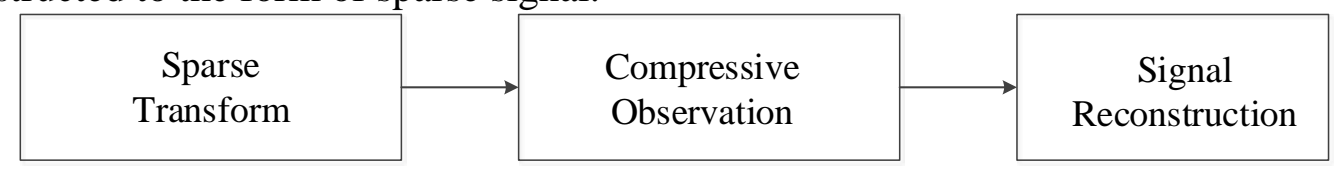

Fig. 1. The algorithm flow graph of delay estimation based on sparse reconstruction

As the indication of Fig.2, the whole time domain is divided into $N$ parts according to the delay, which is $\tilde{\boldsymbol{\tau}}=\left\{\tilde{\tau}_{0}, \tilde{\tau}_{1}, \cdots, \tilde{\tau}_{N-1}\right\}$. Assume that any delay division $\tilde{\tau}_{l}(l=0,1, \cdots, N-1)$ is corresponded with one potential channel. "•• indicates the existent channel, and "॰" indicates the non-existent channel. So each line of the matrix $\tilde{\boldsymbol{a}}=\left[\tilde{a}_{0} e^{-j 2 \pi f_{c} \tau_{0}}, \tilde{a}_{1} e^{-j 2 \pi f_{c} \tau_{1}}, \cdots, \tilde{a}_{N-1} e^{-j 2 \pi f_{c} \tau_{N-1}}\right]^{\mathrm{T}}$ is corresponded with one potential channel, in order to reflect the sparse degree of the signal in the time domain, the potential multi-channel's quantity shall be much more than the practical multi-channel, that is $N \gg L_{p}$, so that $\boldsymbol{a}$ is the representation matrix of the signal gain coefficient. 


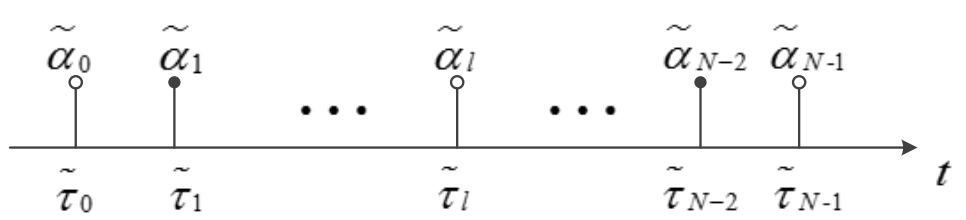

Fig. 2. Sparse representation of the delay in the time domain

Since the steering matrix $\widetilde{\boldsymbol{V}}=\left[\boldsymbol{v}\left(\tilde{\tau}_{0}\right), v\left(\tilde{\tau}_{1}\right), \cdots, v\left(\tilde{\tau}_{N-1}\right)\right]$ includes all information of the delay, so that $\boldsymbol{\Phi}_{0}=\boldsymbol{S} \widetilde{\boldsymbol{V}}$ can be assumed as the measurement matrix.

$$
\left\{\begin{array}{l}
\hat{\boldsymbol{a}}=\arg \min \|\tilde{\boldsymbol{a}}\|_{0} \\
\left\|\boldsymbol{Y}-\Phi_{0} \tilde{\boldsymbol{a}}\right\|_{2} \leq \sigma
\end{array}\right.
$$

The coefficient sparse vector $\tilde{\boldsymbol{a}}$ is reconstructed accurately based on the observed value of $\boldsymbol{Y}=\boldsymbol{\Phi}_{0} \tilde{\boldsymbol{a}}+\boldsymbol{N}$ the optimal $\ell_{0}$ norm question of formulas (10), and then the multi-channel delay estimation shall be obtained according to one-to-one correspondence relationship between $\tilde{\boldsymbol{\tau}}$ and $\tilde{\boldsymbol{a}}$.

\section{Estimation Algorithm of Sparse Reconstruction Delay}

For the solution of the formula (10), all $C_{N}^{L_{p}}$ kinds of combination that are nonzero value shall be exhausted in the so that it is not available to solve directly. The column vector (atom) that is matching with the signal optimally can be selected in the measurement matrix $\boldsymbol{\Phi}_{0}$ to solve. The optimal matching atom is that the atom provided with the maximum projection value on the space constructed by atoms. The projection value is the absolute value of the inner product between the signal and each atom, which is:

$$
u=\left\{u_{j}\left|u_{j}=\right|\left\langle\boldsymbol{Y}, \boldsymbol{\Phi}_{0_{j}}\right\rangle \mid, j=1,2, \cdots N\right\}
$$

Therefore, the norm question solution of the optimal $\ell_{0}$ can be converted to the solution of linear programming question.

\section{Measurement Matrix}

That the necessary and sufficient condition of the coefficient sparse matrix is reconstructed successfully by the measurement matrix is the isometry criterion of the measurement matrix meeting the constraint, which is deduced by the literature [11]. For the sparse signal $\tilde{\boldsymbol{a}}$ of with sparse degree of $L_{p}$, the definition of RIP: provided that the inequality $\left(1-\delta_{L_{p}}\right)\|\hat{\boldsymbol{a}}\|_{2}^{2} \leq\left\|\boldsymbol{\Phi}_{0} \hat{\boldsymbol{a}}\right\|_{2}^{2} \leq\left(1+\delta_{L_{p}}\right)\|\hat{\boldsymbol{a}}\|_{2}^{2}$ is satisfied, if $0<\delta_{L_{p}}<1, \boldsymbol{\Phi}_{0}$ is the linear operator taking parameter $\delta_{L_{p}}$ to meet RIP criterion. To prove whether a determined matrix $\boldsymbol{\Phi}_{0}$ meets the RIP criterion is a combination question, and it is impractical to calculate by using all combinations $\delta_{L_{p}}$ when value $L_{p}$ is high. Candès and other people proved that the RIP criterion can be described equivalently as: if any $2 L_{p}$ of $\boldsymbol{\Phi}_{0}$ is unrelated with the linearity, $\boldsymbol{\Phi}_{0}$ satisfies the RIP criterion. 


$$
\begin{aligned}
\boldsymbol{\Phi}_{0} & =\boldsymbol{S} \cdot \tilde{\boldsymbol{V}} \\
\boldsymbol{\Phi}_{0} & =\left[\begin{array}{lll}
s(0) & \cdots & s(0) \\
s(1) e^{-j 2 \pi \Delta f \tau_{0}} & \vdots & s(1) e^{-j 2 \pi \Delta f \tau_{N-1}} \\
\vdots & \ddots & \vdots \\
s(K-1) e^{-j 2 \pi \Delta f(K-1) \tau_{0}} & \cdots & s(K-1) e^{-j 2 \pi \Delta f(K-1) \tau_{N-1}}
\end{array}\right]
\end{aligned}
$$

Assuming $a_{1} \boldsymbol{\Phi}_{0_{1}}+a_{2} \boldsymbol{\Phi}_{0_{2}}+\cdots+a_{2 L_{p}} \boldsymbol{\Phi}_{0_{2 L_{p}}}=0$, since the rows of $\widetilde{\boldsymbol{V}}$ are provided with similar structure with coefficient difference of $e$ index only, if the above formula is required to satisfy, only assume $a_{1}=a_{2}=\cdots=a_{2 L_{p}}=0$, so that any $2 L_{p}$ row of $\boldsymbol{\Phi}_{0}$ is unrelated with the linearity, and $\boldsymbol{\Phi}_{0}$ satisfies the RIP criterion.

\section{Reconstruction Algorithm}

Reconstruct the signal by using the OMP algorithm, which the algorithm shall select the atom from the measurement matrix, which is matched with the signal in maximum range, and then sparsely approach and work out the surplus, finally the signal shall be indicated in linearity by selected atom after the iteration. Since the OMP algorithm only selects the atom with the maximum projection value by the signal in the constructed space by atoms, so the atom that is provided with several projection value will occur at the same time in the condition of the sparse degree less than 1, so that it will select the suboptimum atom.

The ROMP algorithm is evolved from OMP. As one of the greedy algorithms, ROMP algorithm is simple and easy to realize. At the same time, several atoms that meet the certain conditions are joined instead of only one atom. For $K$ sparse signal, after $K$ iterations, we can estimate the value of delay of the signal in a high precision. Therefore, the computation is further reduced compared with the OMP algorithm. Furthermore, another advantage of the ROMP algorithm is that the reconstruction accuracy is higher than that of the OMP algorithm.

In the compressed-sensing sparse-reconstruction algorithm, the most important thing is to determine which subspace that received signal $y$ is located in. These subspaces are generated by the atoms in the measurement matrix $\boldsymbol{\Phi}_{0}$. Once the correct subspace is determined, the nonzero coefficients of $a$ can be calculated by using the pseudo inverse of the subspace. An important feature of CS-SP algorithm is to find a method to generate $K$ atoms of the correct subspace. The algorithm consists of $K$ atoms of the list in $\boldsymbol{\Phi}_{0}$, the initial estimation of the subspace is the $K$ atoms which get the largest $u$ values according to the formula (14). In order to correct the initial estimation of the subspace, CS-SP algorithm will detect whether the existing subspace can reconstruct the signal in an ideal error. If not, the subspace will be updated. The rule of updating is that the residual of the signal reconstructed by the updated-subspace is lower than that by the not-updated-subspace. CS-SP can ensure that a better subspace can be found in the next iteration based on the above conditions.

The subspace pursuit delay estimation algorithm flow chart is as follows:

1. Input: Sparsity $K$; Measurement matrix $\Phi_{0} ;$ Received signal $y$.

2. Initialization: Use the formula (11) to calculate the projection value $u$, and select $K$ atoms which have the maximum values of $u$. Set the initial subspace $T_{0}$ with these $K$ atoms. 
3. Calculate the residual. $y_{r}^{0}=\operatorname{resid}\left(\mathrm{y}, \Phi_{T_{0}}\right)$

4. Iteration: Extend the subspace. $T_{m}=T_{m-1} \cup\{$ the largest $K$ atoms corresponded with $\left.\widetilde{\Phi} * y_{r}^{m-1}\right\}$

5. Reconstruct the signal. $x_{p}=\widetilde{\Phi}_{T_{l}}^{\dagger} y$

6. Update the subspace. $T_{m}=T_{m-1} \cup\{K$ indices corresponding to the largest element of $\left.x_{p}\right\}$

7. Update and compare the residual. $y_{r}^{m}=\operatorname{resid}\left(\mathrm{y}, \Phi_{T_{m}}\right)$, quit the iteration and let $T_{m}=T_{m-1}$.

8. Output: The reconstructed signal $\bar{x}$.

\section{Performance Simulation}

The article here conduct study for the delay estimation algorithm in the wireless location model, and the OFDM signal is planned to be used as the sending signal. The system parameters are set as Table 1 .

Table 1. System Parameters Settings

\begin{tabular}{|c|c|}
\hline \multicolumn{2}{|c|}{ System Parameters } \\
\hline Subcarrier wave number & 64 \\
\hline Carrier frequency & $2.4 \mathrm{GHz}$ \\
\hline System band width & $20 \mathrm{MHz}$ \\
\hline Intervals & $0.2 \mathrm{MHz}$ \\
\hline
\end{tabular}

In order to verify the practicability and the robustness of the algorithm applied in this article, the Monte Carlo experiment shall be conducted to compare and analyze the CS-SP algorithm, OMP algorithm and ROMP algorithm.

Firstly the signal-to-noise ratio can be defined as:

$$
S N R=10 \lg \frac{|y(t)|^{2}}{|n(t)|^{2}}
$$

In the formula (14), $y(t)$ is the received signal, and $n(t)$ is the additive Gauss white noise.

Mean square error is:

$$
\operatorname{RMSE}_{i}=\sqrt{\frac{1}{M}\left(\sum_{m=1}^{M}\left|\hat{\tau}_{m, i}-\tau_{i}\right|^{2}\right)}, i=1,2, \cdots, L_{p}
$$

In (15), $M$ is the Monte Carlo simulation times; $\hat{\tau}_{m, i}$ is the delay estimation of the No. i channel's No. $m$ time of Monte Carlo experiment; $\hat{\tau}_{i}$ is the No. $i$ channel's true value of the delay.

\section{Simulation 1}

It is supposed that the arrival time of multi-channel received signal's channels quantity (sparsities) $L_{p}=2$ are separately $\tau_{1}=100 \mathrm{~ns}$ and $\tau_{2}=700 \mathrm{~ns}$, with snapshot number of 1 . If $\mathrm{SNR}=25 \mathrm{~dB}$, conduct Monte Carlo simulation with $\mathrm{M}=200$ by the algorithm proposed in this article, which the distribution diagram of the obtained delay estimation value is 
showed in the Fig.3. In the figure, the two squares represent the local amplification values of the delay estimation. According to the Fig.3, the CS-SP algorithm applied in this article is able to achieve unbiased estimation in the condition of single-snapshot.

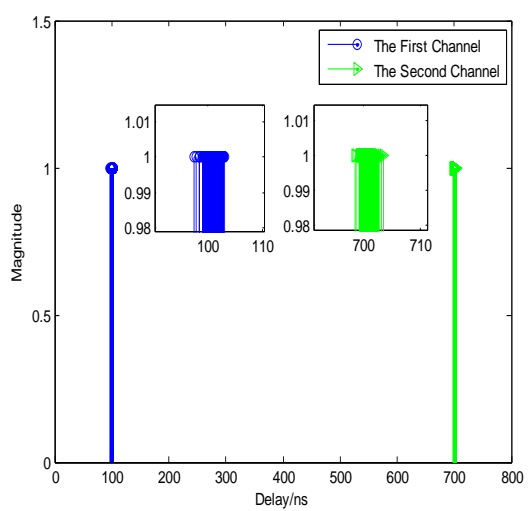

Fig. 3. $\mathrm{SNR}=25 \mathrm{~dB}$, the distribution diagram of the delay estimation in the condition of $L_{p}=2$

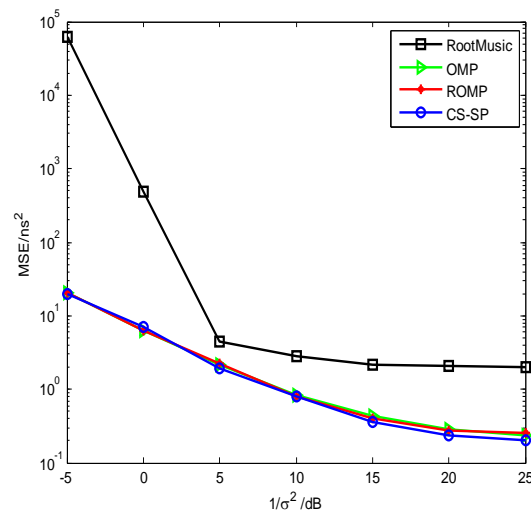

Fig. 4. Contrast diagram of mean square error for various algorithms

\section{Simulation 2}

Under the identical conditions, the CS-SP algorithm shall be compared with OMP algorithm and ROMP algorithm. The mean square error curves of the above algorithms shall be made. The curves are indicated in Fig.4. According to this diagram, in the condition of single-snapshot, the sparse reconstruction method can achieve a better performance compared to existing estimation approaches such as the RootMusic algorithm, since the sparse reconstruction method only needs to collect a single snapshot data, by using the $1_{0}$ norm sparse constraint, and then get the delay estimation values through the reconstruction algorithms. The RootMusic algorithm can hardly achieve an effective estimation in the single snapshot environment. The estimation performance of the CS-SP algorithm is obviously better than the greedy algorithms such as OMP and ROMP. Since the CS-SP algorithm update the subspace where the signal is located in, so that its performance is better than greedy algorithms. Therefore, the accuracy and the robustness of the delay estimation performance of the CS-SP algorithm is better than the OMP and ROMP algorithm.

\section{Simulation 3}

The CS-SP, OMP and ROMP algorithms shall be simulated in various multi-channel quantity (sparsity), and the result is indicated in Fig.5. Now define the exact reconstruction rate is the ratio of the numbers of the accurate estimated channels to the numbers of the real channels, which is decreasing following the increase of sparsities. The CS-SP algorithm can achieve a better anti-multi-channel performance according to the diagram. 


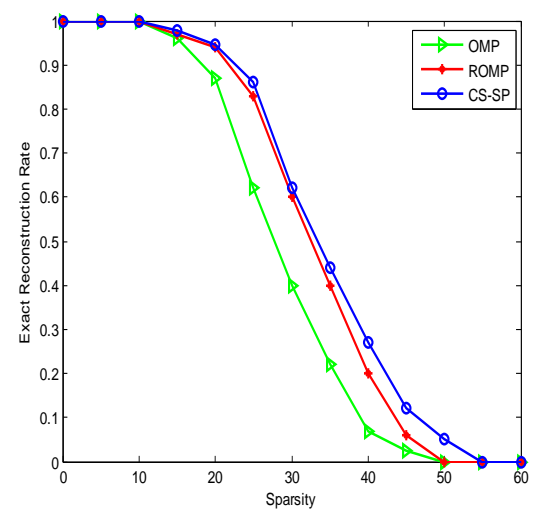

Fig. 5. Exact reconstruction rate in different sparsities

\section{Conclusions}

In the wireless location system, the current sparse reconstruction delay estimation method is provided with better estimation performance in the conditions of single-snapshot and low signal-to-noise ratio, but it has a certain probability to select suboptimum atom in the process of atom selection. For this problem, the CS-SP algorithm is applied in the article. The CS-SP algorithm can decrease the disadvantage in choosing atoms by updating the subspace that the signal is located in, which the estimation performance is better than algorithms of OMP and ROMP in the condition of low signal-to-noise ratio. Comparing with the OMP and ROMP algorithms, the exact reconstruction rate is higher in the high value of sparsities, which means the CS-SP algorithm has a better performance in anti-multi-channel. The simulation result proves that the algorithm is stable, reliable and excellent.

\section{References}

[1] Min Zhang, Yu-Lan Ma, and Bang-Qing Li, Novel loop-like solitons for the generalized Vakhnenko equation (In Chinese), Chinese Physics B,2013,03:275-278.

[2] Fang-qiu Wang, Xiao-fei Zhang, Fei Wang, Root-MUSIC-based joint TOA and DOA estimation in IR-UWB (In Chinese), Journal on Communications,vol.35, NO.2,February 2014.

[3] Jing Li, Jian-dong Zhu, Zhi-hong Feng, Yong-jun Zhao, Dong-hai Li, Passive Multipath Time Delay Estimation Using MCMC Methods, Circuits Syst Signal Process,(2015) 34:3897-3913 DOI 10.1007/s00034-015-0037-1.

[4] David L. Donoho, Compressed Sensing, IEEE TRANSACTIONS ON INFORMATION THEORY, VOL. 52, NO. 4, APRIL 2006.

[5] Hai-tao Wang, Jun Wang, Super-resolution DOA Estimation in Passive Radar Based on Compressed Sensing (In Chinese), Journal of Electronics \& Information Technology, vol. 85, No.4, Apr.2013.

[6] Bo Lin, Zeng-hui Zhang, Ju-bo Zhu, Sparsity Model and Performance Analysis of DOA Estimation with Compressive Sensing (In Chinese), Journal of Electronics \& Information Technology, vol. 36, No.3, Mar.2014. 
[7] Yu-feng Chen, Jian-guo Huang, Jian-jun Su, High Resolution Direction-of-Arrive Estimation Based on Sparse Reconstruction and Compressive Sensing Beamforming (In Chinese), TORPEDO TECHNOLOGY, vol. 21, No.2, Apr.2013.

[8] Fang-qiu Wang and Xiao-fei Zhang, Joint Estimation of TOA and DOA in IR-UWB System Using Sparse Representation Framework, ETRI Journal, vol. 36, No.3, June 2014.

[9] Jian Yang, Ting Jiang, Cheng-lin Zhao, Zheng Zhou, UWB Channel Estimation Based on CS-ROMP Algorithm, Radio Engineering, 2011,05:14-17.

[10]Wei Dai and Olgica Milenkovic, Subspace Pursuit for Compressive Sensing Signal Reconstruction, Introduction to occupational epidemiology, 1287-1305.

[11]Emmanuel J. Candes and Terence Tao, Decoding by Linear Programming, IEEE TRANSACTIONS ON INFORMATION THEORY, VOL. 51, NO. 12, DECEMBER 2005. 